\title{
XI. Internationaler Ophthalmologen-Kongress in Neapel
}

Der XL internationale Ophthalmologen-Kongress wird unter dem Ehren-Präsidium von Marc Dufour und E. Landolt vom 2.-7. IV. 1909 in Neapel tagen.

Da den ïeilnehmern des Kongresses in Italien sowohl für die Eisenbalm wie für die Sckiffsreise erhebliclie Preiserinässigungön gewährt werden, empfiehlt es sicli, zwecks rechtzeitiger Erlangung der Mitgliedskarten mög-lielist bald die Mitgliedschaft zu bewirken.

Die Sitzungon des Kongresses werden im Königl. Universitäsgebäude, die mit dem Kongresse vorbundene Ausstellung von wissenschaftlichen Apparaten, Instrumenten, Lehnnitteln, Objekten von praktischer wie von kistorischer Bedeutung etc. in den Räumen der Königl. Augenklinik in S. Andrea delle Dame stattfinden. Anmeldungen hierfür sind an den Präsi-denten des Organisationskomitees, Professor Arnaldo Angelucoi, S. Andrea delle Dame, zu richten. Aus Anlass des Kongresses wird das Präsidium, um das Andenken des Gründors der Augenklinik zu Neapel, Professor de Vicentiis, zu ehren, aus dem Fondsrest der internationalen Subskription von 1905 seeks goldene Medaillen den seeks hervorragendsten imveröffentlichten Berichten junger Ophthalmologen zuerkonnen. Diese Medaillen werden den Namen ,Premio de Vicentiis" tragen. Drei derselbon sind für Mitteilungen von Ausländern und drei für solche von Italienorn bestimmt.

Bemerkt sei, dass vom 30. III. bis zum 3. IV. 1909 gleichfalls in Neapel der internationale Kongress für Typhlologie tagen wird. Derselbe wird eine Naelimittagssitzung anberaumen, zu der die Mitglieder des opkthalmologi-sehen Kongresses eingeladen sind, um an der Beratung des Thomas teilzu-nehmen: , Ist es znlässig, solchen Patienten, die mit sicker zur Erblindung führenden Leiden behaftet sind, eine der vollen Walirheit entsprechende Prognose zu geben, damit sick dieselben rechtzeitig, d. h. nock im Besitze eines gewissen Sehvermögons, auf die wäkrend der späteren Blindkeit zu verrichtenden Beschäftigungen vorbereiten können ? Zur Vormittelung der Mitgliedskarten sowie zur Erteilung weiterer Aus-künfte ist der Korrespondent für Norddeiitschland, Professor Kuhnt-Honn, jederzeit gern bereit.

Die neue Universitäts-Augenklinik in Budapest.

Die mit alienVerbesserungen und Hülfsmitteln derNeuzeit ausgestattete Universitätsaugenklinik in Budapest, in welcher die opkthalmologisch $\theta$ Sektion des internationalen medizinischen Kongresses im August dieses Jahres tagen wird, ist am 1. IX. 1908 vom Direktor Professor Emil v. Grosz eröffnet worden. Die 70 Meter lango Vorderfront des überaus statt-licken Gebäudes ist nack Südosten gelegen und von der Strasse durch einen 4 Motor breiten Vorgarten getrennt. Im Untergesckoss befinden sicli die Woknungen des Pförtners, Heizers, Dieners etc., ferner der Kesselraum, die Wasckkücke, die Unterkunft für die Versuckstiere, die Räume für die Desinfektion etc. etc.

94 Literatur-Verzeichnis.

Im Erdgeschoss $\beta$ sind die Untersuchungsräume: der Hörsaal, die Kurssäl $\beta$, die Laboratorien, die Bibliothek sowie die ßäume für dio Poli-klinik, die einen besonderen Eingang haben, imtergebracht. 
Das erste Stookwerk enthält die Zimmer für die nicht operierten, das zweite diejenigen für die operierten Kranken. In letzterem sind ausserdem gelegen der Operationssaal mit Sterüisationsraum, das Vorbereitungs- das Instrumenten- und das Verbandzimmer.

Ein Aufbau in der Mitte des Gebäudes beherbergt die Wohnungen der Assistenten, das photographische Atelier etc.

Die Beheizung gesehieht durch eine Warmwasser-Heiz-Anlage. Der Fussboden ist in den poliklinischen Räumen mit Platten, in den Kranken-und Wohnräumen mit in Asphalt eingelegtem Hartholz, im Hörsaal mit linoleum belegt. Die Beleuchtung ist eine ausschliesslieh elektrische. Ein Fahrstuhl geht vom Erd- bis zum Dachgeschoss.

Die Zahl der Krankenbetten ist auf 80 festgesetzt. Drei Assistenten, drei Assistenzärzte, eine Oberwärterin, 10 Wärterinnen bilden das Aerzte-und Pflegepersonal.

Das Inventar der alten Klinik eingerechnet (welches auf 35000 Kronen zu veranschlagen ist) belaufen sich die Gesamtkosten auf 900000 Kronen gleich 750000 Mark. 